\title{
Flexibility Assesment of Fully Inkjet-Printed Reconfigurable Antenna With $\mathrm{VO}_{2}$ Switch
}

\author{
Mohammad Vaseem, Zhen Su, Shuai Yang, Atif Shamim \\ King Abdullah University of Science and Technology (KAUST), IMPACT Lab, Computer, Electrical and Mathematical Sciences and \\ Engineering (CEMSE) Division \\ Thuwal 23955-6900, Kingdom of Saudi Arabia \\ mohammad.vaseem@kaust.edu.sa,su.zhen@kaust.edu.sa, shuai.yang@kaust.edu.sa, atif.shamim@kaust.edu.sa
}

\begin{abstract}
With the surge of Internet of Things (IoT) applications, billions of wireless devices need to be manufactured which could be mounted on non-planar objects or be worn by humans. This is where printing flexible electronics can be a game changer. Recently, frequency-reconfigurable radio-frequency (RF) components are in high demand due to multiple frequencybands in wireless devices. A very important part of reconfigurable components is RF switch. Traditional switches are based on PIN diode, microelectromechanical-systems (MEMS), transistor, ferrite- and ferro-electric based devices. All these traditional switches have their own sets of advantages and disadvantages, however, one issue is common, almost all RF switches need to be soldered or attached to the reconfigurable components. Thus, it is hard to maintain switch configuration in flexible condition. To overcome these issues, we demonstrate a printing technique to fabricate reconfigurable antenna supported on flexible kapton substrate with switch based on $\mathrm{VO}_{2}$ ink. The fully printed antenna operates at $2.5-3.5 \mathrm{GHz}$ when the switch is in the OFF state. When switch is thermally activated in the ON state, it operates at 1.6-2.6 GHz. The printed antenna shows a maximum gain of $\sim 2 \mathrm{dBi}$ at $3.2 \mathrm{GHz}$ with concave bending position. Antenna performance in different bent configurations supports its uses for many RF designs that require reconfigurability and tunability.
\end{abstract}

Keywords - bending test, inkjet-printing, phase change, reconfigurable-antenna, vanadium dioxide,

\section{INTRODUCTION}

In the wireless industry, there is a big desire to have tunable and re-configurable radio-frequency (RF) components (such as antenna and filters) which are required to cover multiple frequency bands or to be able to tune to a different wireless standard in a different zone of the world. This means that an antenna designed for Asian frequency bands may not work for the North American bands. Thus, there is a requirement that these antennas or filters can be tuned or reconfigured to different bands for different standards. The utilization of tunable and reconfigurable devices in such system can effectively decrease the complexity of the design and reduce the size of the system, and ultimately brings down the cost. The core of the tunability and reconfigurability of the devices are the RF switches, which is capable of blocking or allowing RF signals to pass through it based on applied stimuli. In addition to the multiband requirement, flexibility and bending support is required in many cases.

Several kinds of technologies are used for RF-switching applications, such as ferrite- and ferro-electric based devices [1], a transistor-based switch [2], a microelectromechanical- systems-based (MEMS-based) [3], PIN diode-based switch [4], and so on. The most important disadvantages of these available switches is that they have been realized through expensive and complicated fabrication protocols.

Recently, vanadium dioxide $\left(\mathrm{VO}_{2}\right)$ based phase-change material has been recognized as interesting alternatives for switching applications. The electrical properties of $\mathrm{VO}_{2}$ can be triggered with current or heat [5]. Vanadium dioxide typically exhibits metal-insulator transition (MIT) in a reversible fashion at a temperature of $68{ }^{\circ} \mathrm{C}$. Thus, $\mathrm{VO}_{2}$ offered a favorable material for reconfigurable devices and high-speed switching. There are few reports where RF switches using $\mathrm{VO}_{2}$ material is demonstrated but all of these devices have been fabricated using nanofabrication process and also with high vacuum pressure and temperature based deposition techniques such as sputter, MOMBE, PLD or evaporation [5-7].

In this work, fully printed antenna with frequencyreconfigurability is demonstrated by printed $\mathrm{VO}_{2}$ switch supported on a flexible Kapton substrate. The printed antenna operates at $2.4 \mathrm{GHz}$ when $\mathrm{VO}_{2}$ switch is in $\mathrm{ON}$ state and when it is in OFF state, it operates at $3.5 \mathrm{GHz}$. The antenna performance is also evaluated with different bending condition such as in concave and convex bent configurations. The antenna is shown steady performance at different bend positions which further confirms its mode of operation on flexible substrates.

\section{MATERIALS AND FABRICATION}
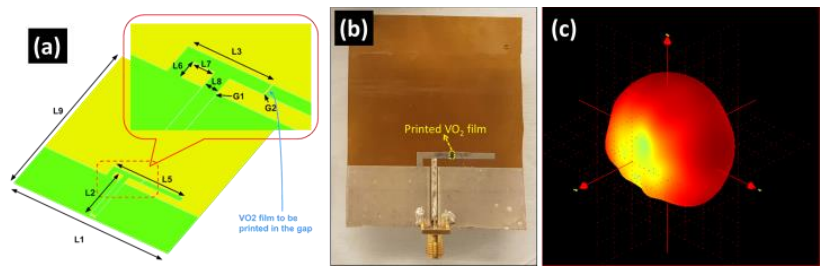

Fig. 1. (a) A 3D depiction of as-proposed PIFA antenna, (b) camera image of fabricated prototype and (c) its corresponding measured radiation pattern@ 3.2 $\mathrm{GHz}$ in OFF state. The dimension in (a) is as follows: $\mathrm{L} 1=60,000 \mu \mathrm{m}, \mathrm{L} 2=$ $21,000 \mu \mathrm{m}, \mathrm{L} 3=11,800 \mu \mathrm{m}, \mathrm{L} 4=15,000 \mu \mathrm{m}, \mathrm{L} 5=27,000 \mu \mathrm{m}, \mathrm{L} 6=3000$ $\mu \mathrm{m}, \mathrm{L} 7=3000 \mu \mathrm{m}, \mathrm{L} 8=2000 \mu \mathrm{m}, \mathrm{L} 9=50,000 \mu \mathrm{m}, \mathrm{G} 1=53 \mu \mathrm{m}, \mathrm{G} 2=200$ $\mu \mathrm{m}$.

$\mathrm{VO}_{2}$ is prepared in the form of nanoparticles by a simple solution process. Typically, $\mathrm{VO}_{2}$ material has many crystalline structure phases, however, the preferable phase is monoclinic $\mathrm{VO}_{2}(\mathrm{M})$ which has the ability to show phase transition at 68 ${ }^{0} \mathrm{C}$. To obtain the $\mathrm{VO}_{2}(\mathrm{M})$ phase, as-prepared nanoparticles were optimized with annealing condition. For the ink- 
formulation, annealed $\mathrm{VO}_{2}$ nanoparticles were treated with oleic acid to make them compatible with organic solvents. After successful treatment with oleic acid, the $\mathrm{VO}_{2}$ nanoparticles were dispersed in the solvent mixture of 2methoxy ethanol, chlorobenzene and ethanol with volumetric ratio of $3.5,0.3$, and $0.2 \mathrm{ml}$, respectively. The design of the antenna is shown in Fig. 1 (a). In the antenna design, frequency-reconfigurability is proposed by extruding the arm length, labeled as L5 (L5 = L3 + L4). Since the working frequency is inversely proportional to the length of L5, for a longer arm (L5) the antenna works at a lower frequency. In contrast, a shortened arm with length L3 shifts the antenna to a higher operating frequency. For the fabrication of PIFA antenna, a total of 8-layers of SOC ink [8] were printed and cured on the flexible Kapton substrate. Afterwards, $\mathrm{VO}_{2}$ switch is printed on the gap area $(200 \mu \mathrm{m} \times 2000 \mu \mathrm{m}$ square $)$. After $\mathrm{VO}_{2}$ printing, the printed-film is cured at $200{ }^{\circ} \mathrm{C}$ in the vacuum for $1 \mathrm{~h}$. After curing process, SMA connector is mounted with silver epoxy. The final prototype is shown in Fig. 1 (b).

\section{BENDING TEST ON ANTENNA WITH SWITCH}

In order to analyze the antenna performance with different bending condition, the printed antenna is bent with convex and concave configuration with 20 and $40 \mathrm{~mm}$ bending followed by radiation pattern assessment, as shown in Fig. 2. The reflection coefficient of the antenna with flat condition is shown in Fig 3 (a) which is confirmed the frequency band of 2.57-3.50 GHz when the switch is at "OFF" state $(\mathrm{R}=\sim 1.2$ $\mathrm{k} \Omega)$. However, when the switch is at "ON" state $(\mathrm{R}=\sim 10 \Omega)$, the frequency is shifted to lower band of 1.65-2.60 GHz. The effect of the bending to its starting frequency is very minimal, there is no change in both $20 \mathrm{~mm}$ bending cases, but a maximum of $20 \mathrm{MHz}$ shift is observed with $40 \mathrm{~mm}$ bending. The stop frequencies have a maximum shift of $200 \mathrm{MHz}$ in all cases except concave bending of $40 \mathrm{~mm}$. Even with a noticeable shift, in all the bending cases the antenna still match with its input impedance at its desired frequency bands at both $\mathrm{ON}$ and $\mathrm{OFF}$ states. The radiation patterns in the bent configurations are also measured for antenna in OFF state, asshown in Fig. 2 and summarized in Table I. In case of the concave bending of $20 \mathrm{~mm}$, maximum gain of $2 \mathrm{~dB}$ is observed in the frequency band at $3.2 \mathrm{GHz}$ due to slight increase in the directivity when antenna is bent. However, the gain is further reduced with convex and $40 \mathrm{~mm}$ concave bending.

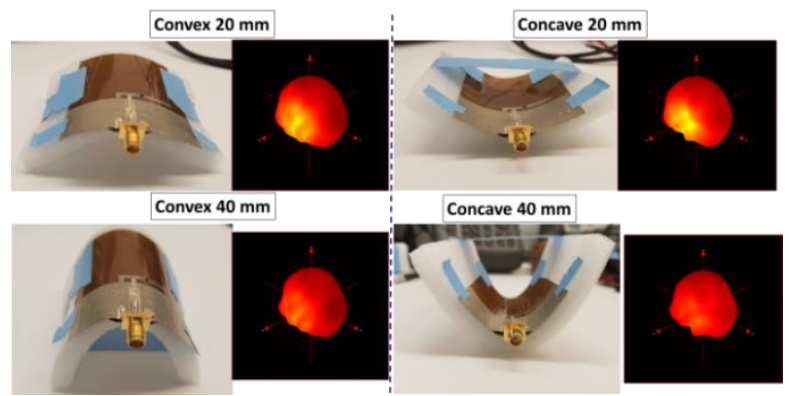

Fig. 2. Fabricated antenna with different bending condition and its corresponding measured radiation patterns @ 3.2 GHz in OFF state (a)
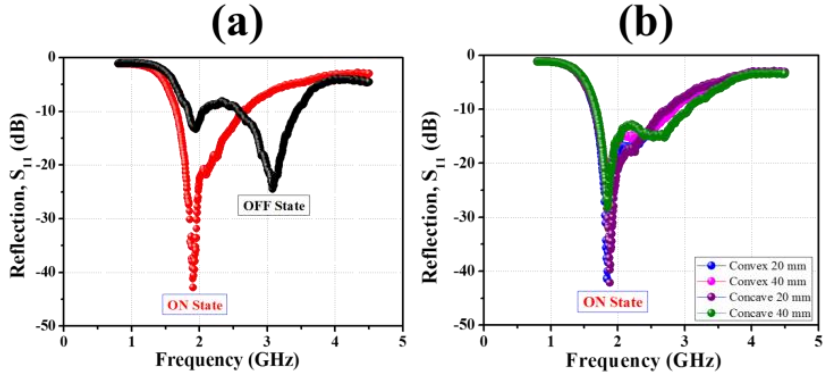

Fig. 3. The $\mathrm{S}_{11}$ of antenna with $\mathrm{VO}_{2}$ switch in ON/OFF state at (a) flat condition and (b) different bending condition

TABLE I. MEASURED ANTENNA GAIN COMPARSION WITH SWITCH IN OFF STATE AT DIFFERENT BENDING CONDITION

\begin{tabular}{|c|c|c|c|c|c|}
\hline $\begin{array}{c}\text { Frequency } \\
(\mathbf{G H z})\end{array}$ & Flat & $\begin{array}{c}\text { Convex } \\
\mathbf{( 2 0} \mathbf{~ m m})\end{array}$ & $\begin{array}{c}\text { Concave } \\
\mathbf{( 2 0} \mathbf{~ m m})\end{array}$ & $\begin{array}{c}\text { Convex } \\
(\mathbf{4 0} \mathbf{~ m m})\end{array}$ & $\begin{array}{c}\text { Concave } \\
\mathbf{( 4 0} \mathbf{~ m m})\end{array}$ \\
\hline $\mathbf{2 . 4}$ & 1.49 & 1.53 & 1.70 & 0.94 & 1.31 \\
\hline $\mathbf{3 . 2}$ & 2.11 & 1.68 & 2.07 & 1.33 & 1.33 \\
\hline $\mathbf{3 . 3}$ & 1.83 & 1.60 & 1.90 & 1.28 & 1.19 \\
\hline $\mathbf{3 . 5}$ & 1.24 & 0.93 & 1.41 & 0.53 & 0.90 \\
\hline
\end{tabular}

\section{CONCULSION}

In this report, we presented a novel $\mathrm{VO}_{2}$ nanoparticles based reconfigurable PIFA antenna wherein $\mathrm{VO}_{2}$ printed film is thermally tuned. The antenna is able to switch between two bands with printed $\mathrm{VO}_{2}$ film in ON/OFF state. The overall performance does not change much except a few $\mathrm{MHz}$ shift in both bending condition but match well with the frequency band of interest. The successful implementation of fully printed $\mathrm{VO}_{2}$-based switch with conformal design of antenna and its performance in bent configurations proves its uses for many RF designs that require reconfigurability and tunability.

\section{REFERENCES}

[1] M. Vaseem, et. al., "Iron Oxide Nanoparticle-Based Magnetic Ink Development for Fully Printed Tunable Radio-Frequency Devices," Adv. Mater. Technol. vol. 3, pp. 1700242, 2018.

[2] Z. Imane, et. al., "A ku-band distributed SPDT switch in $0.5 \mu \mathrm{m}$ AlGaN/GaN HEMT technology," Microw. Opt. Technol. Lett., vol. 60, pp. 462-465, 2018.

[3] Y. H. Song, et. al., "Complementary Logic Inverters: High-Performance Hybrid Complementary Logic Inverter through Monolithic Integration of a MEMS Switch and an Oxide TFT," Small, vol. 11, pp. 1357-1357, 2015.

[4] U. Gaurav, et. al., "Pin-diode based switchable multiband dual feed microstrip patch antenna," Microw. Opt. Technol. Lett. vol. 59, pp. 14541460, 2017.

[5] F. Dumas-Bouchiat, et. al., "rf-microwave switches based on reversible semiconductor-metal transition of $\mathrm{VO}_{2}$ thin films synthesized by pulsedlaser deposition," Appl. Phys. Lett., vol. 91, no.6, pp.223305-223307, 2007.

[6] K. Pan, et. al., "Vanadium Oxide Thin-Film Variable Resistor-Based RF Switches," IEEE Trans. Electron Devices vol. 62, pp. 2959-2965, 2015.

[7] T. S. Teeslink, et. al. "Reconfigurable Bowtie Antenna Using MetalInsulator Transition in Vanadium Dioxide", IEEE Antennas \& Wireless Propagation Lett., vol.14, pp.1381-1384, 2015.

[8] M. Vaseem, et al., "Robust Design of a Particle-Free Silver-OrganoComplex Ink with High Conductivity and Inkjet Stability for Flexible Electronics," ACS Appl. Mater. \& Interfaces, vol. 8, pp 177-186, 2015. 